Provided for non-commercial research and education use. Not for reproduction, distribution or commercial use.

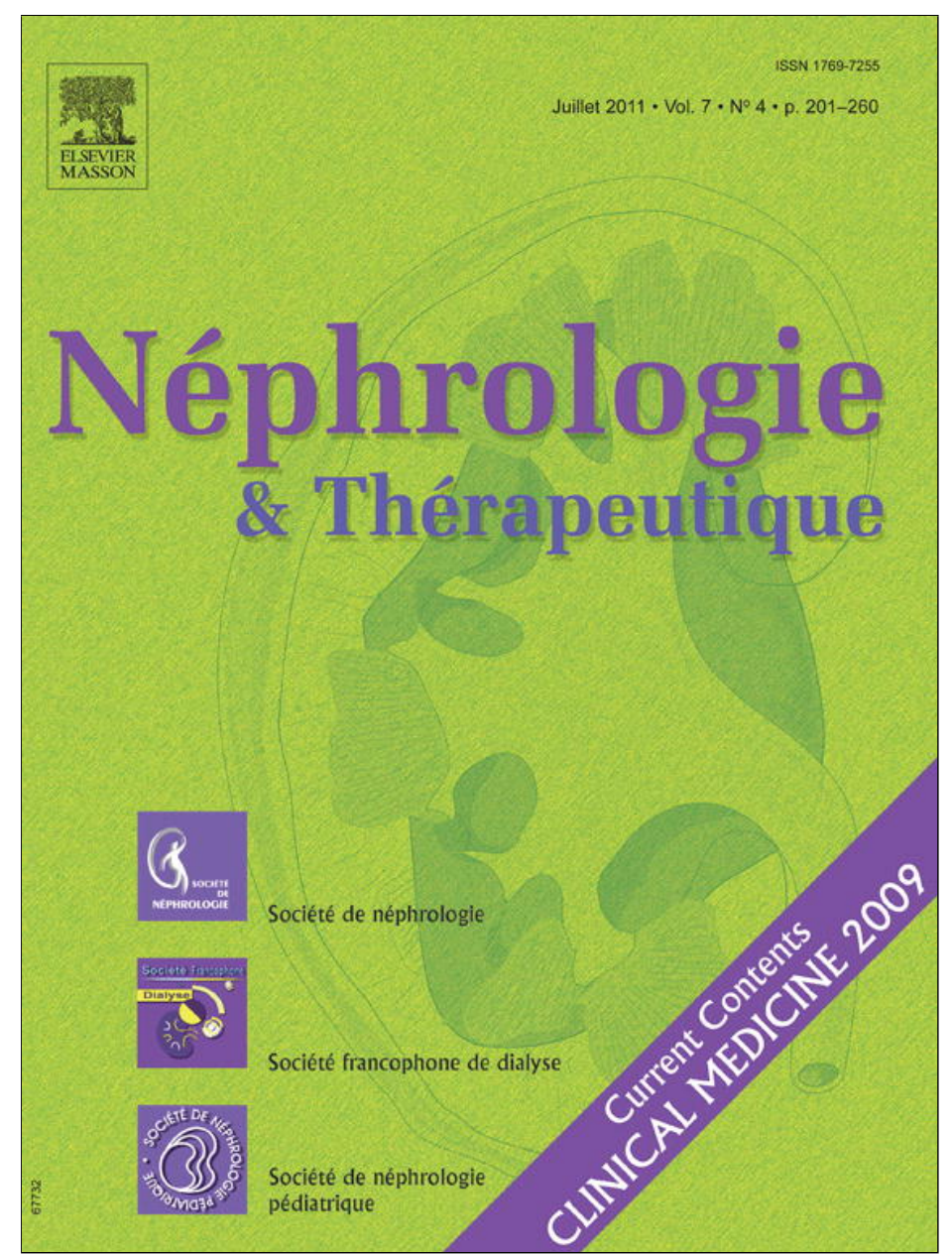

This article appeared in a journal published by Elsevier. The attached copy is furnished to the author for internal non-commercial research and education use, including for instruction at the authors institution and sharing with colleagues.

Other uses, including reproduction and distribution, or selling or licensing copies, or posting to personal, institutional or third party websites are prohibited.

In most cases authors are permitted to post their version of the article (e.g. in Word or Tex form) to their personal website or institutional repository. Authors requiring further information regarding Elsevier's archiving and manuscript policies are encouraged to visit:

http://www.elsevier.com/copyright 
Article original

\section{Intérêt des interventions psychologiques en dialyse : étude exploratoire}

\section{Interest of psychological interventions in dialysis: Exploratory study

\author{
C. Rocha Augusto a,*, J.-M. Krzesinski ${ }^{\text {b }}$, X. Warling ${ }^{\text {c }}$, N. Smelten ${ }^{\text {d }}$, A.-M. Étienne ${ }^{a}$
}

a Service de psychologie de la santé, département personne et société, faculté de psychologie de l'université de Liège, bâtiment B33, Trifac, 3, boulevard du Rectorat, 4000 Liège, France

${ }^{\mathrm{b}}$ Service de néphrologie du centre hospitalier universitaire de Liège, bâtiment B35, Tour 1, 6 é étage, boulevard de l'Hôpital, 4000 Liège, France

${ }^{\mathrm{c}}$ Service de néphrologie du centre hospitalier régional de la Citadelle, 1, boulevard du 12 de ligne, 4000 Liège, France

${ }^{\mathrm{d}}$ Service de néphrologie du centre hospitalier du Bois-de-l'Abbaye-et-de-Hesbaye, 40, rue Laplace, 4100 Seraing, France

\section{N F O A R T I C L E}

\section{Historique de l'article}

Reçu le 18 janvier 2010

Accepté le 11 décembre 2010

\section{Mots clés}

Dialyse

Maladies rénales

Anxiété

Dépression

Qualité de vie

Interventions psychologiques

\section{R É S U M É}

Les patients souffrant d'une insuffisance rénale chronique (IRC) ont une qualité de vie fortement réduite accompagnée d'une détresse émotionnelle sévère (inquiétudes-anxiété-dépression élevée). Pourtant, en Belgique, aucune aide psychologique régulière n'est proposée aux patients dialysés. Notre objectif est de montrer qu'une intervention psychologique peut diminuer significativement la détresse émotionnelle des patients IRC. Onze séances d'interventions structurées sont proposées aux patients IRC sélectionnés selon certains critères : être majeurs, ne pas présenter de confusion ou de démence, être en hémodialyse depuis au moins trois mois, avoir obtenu 14 ou plus à l'échelle HAD. Les interventions portent sur la gestion des symptômes anxieux et dépressifs ainsi que de la maladie elle-même. Trois modules d'interventions indépendants ont ainsi été constitués. Des questionnaires sont remplis par les patients à différentes étapes pour évaluer l'anxiété et la dépression (HADS), les inquiétudes (Penn State) et la qualité de vie (KDQoL-SF). Les résultats observés pour les 47 patients montrent une diminution significative des scores d'anxiété, de dépression et d'inquiétudes ainsi qu'une amélioration significative de la qualité de vie. Parallèlement, nous avons constaté une diminution significative du produit biologique calcium-phosphore obtenu avant dialyse.

(c) 2011 Association Société de néphrologie. Publié par Elsevier Masson SAS. Tous droits réservés.

A B S T R A C T

Patients suffering from end-stage renal disease (ESRD) have a very reduced quality of life accompanied by a severe emotional distress (high worries-anxiety-depression). However, in Belgium, no regular psychological intervention is proposed to dialyzed patients. Our objective is to show that psychological intervention can significantly decrease the emotional distress of patients with ESRD. Eleven sessions of structured interventions are proposed to ESRD patients. Eligibility criteria are to be major, to not present confusion or/and dementia, to have been on dialysis treatment for at least 3 months, to have obtained 14 or more on HAD-scale. Interventions carry on the management of anxious and depressive symptoms and of the disease itself. This constitutes three independent modules. Questionnaires are filled in by the patients at various stages to evaluate the anxiety and the depression (HADS), the worries (Penn State) and the quality of life (KDQoL-SF). Results for the 47 ESRD patients show a significant reduction of the scores of anxiety, depression and worries and a significant growth of quality of life. In parallel, a decrease in the serum calciumphosphorus product analyzed before dialysis has been noted.

(c) 2011 Association Société de néphrologie. Published by Elsevier Masson SAS. All rights reserved.

\footnotetext{
* Auteur correspondant.

Adresse e-mail : rocha_cindy@hotmail.com (C. Rocha Augusto).
}

\section{Introduction}

En Belgique, près d'un patient sur 1000 est touché par une insuffisance rénale terminale (IRT) et se retrouve contraint de recourir à un traitement de suppléance (dialyse) [1]. L'impact de ce type de traitement sur la santé psychologique du patient est 
de plus en plus étudié. Fatigue, douleurs, restrictions hydriques et alimentaires, temps passé en dialyse, conséquences sur la vie sexuelle des patients sont des éléments qui peuvent agir directement sur le moral et la qualité de vie des personnes dialysées [2]. Ainsi, entre 20 et $56,7 \%$ des patients dialysés dans le monde sont dépressifs [3-10] et entre 20 et $45 \%$ présentent un état anxieux [11-14].

En 2008, nous avons confirmé ces données auprès de 133 patients dialysés francophones [15] : 28,8 \% des patients présentent des signes cliniques de dépression et $24,2 \%$ des symptômes d'anxiété. Dans toutes les dimensions évaluées à l'aide du Medical Outcome Study Short Form 36 item health survey (MOS SF-36), les patients atteints d'insuffisance rénale chronique terminale ont une qualité de vie détériorée par rapport à la population générale. Au regard de ces premières données, l'objectif de notre travail sur le terrain a évolué vers le développement de stratégies facilitant, chez le patient, l'acceptation et l'adaptation aux différents changements occasionnés par la maladie rénale terminale. Alors que la littérature scientifique est de plus en plus documentée sur la détresse émotionnelle des patients IRT, très peu d'études ont testé l'impact d'interventions psychologiques structurées destinées aux patients dialysés.

Nous avons répertorié deux types d'études. Dans le premier type d'études, les stratégies présentées visent, avant tout, l'amélioration de l'adhésion thérapeutique des patients. Dans le second type, les auteurs se focalisent sur une amélioration de la détresse émotionnelle.

Les études du premier type soulignent une amélioration de l'adhésion thérapeutique des patients dialysés [16-19] ; la possibilité de retarder au maximum l'entrée en dialyse ainsi qu'un meilleur ajustement entre type de dialyse et patient [2022 ] ; une amélioration de l'autonomie des patients permettant, à terme, de les rediriger vers une antenne d'autodialyse [23] et enfin une amélioration de leur état de santé et de leur qualité de vie globale grâce à un programme de réentraînement sportif [24].

Les deux études centrées sur les programmes d'aide psychologique aux patients sont issues de la même équipe et visent directement l'amélioration de la qualité de vie et la diminution du niveau de dépression des patients $[25,26]$. C'est, selon nous, la seule équipe ayant adopté une perspective bio- psychosociale au sein de la littérature scientifique. Les résultats principaux sont une diminution du stress ressenti, du niveau de dépression ainsi qu'une amélioration de la qualité de vie par rapport au groupe témoin.

En revanche, aucune étude visant à la fois une diminution de la détresse psychologique et une amélioration de l'adhésion aux diverses mesures de santé n'a été réalisée.

C'est la raison pour laquelle notre travail a voulu prendre en compte ces deux dimensions à la fois. Ainsi, notre hypothèse générale est qu'un soutien psychologique structuré issu des thérapies cognitivo-comportementales (TCC) favorisera la diminution de la détresse émotionnelle des patients insuffisants rénaux chroniques (anxiété, inquiétudes et dépression) et améliorera, de manière indirecte la qualité de vie. Par ailleurs, la psychoéducation intégrée dans notre programme provoquera une diminution de la prise de poids interdialytique et une diminution des taux de potassium et du produit calciumphosphore.

Le choix spécifique de notre prise en charge TCC se justifie par les résultats déjà obtenus en dialyse $[17,18,23,25,26]$ mais aussi en cardiologie [27-31], en oncologie [32,33], et en gériatrie [34]. Les techniques principales utilisées sont : la relaxation, la restructuration cognitive et la résolution de problèmes. Plus spécifiquement, une revue systématique de la littérature sur la dépression montre que les groupes recevant des interventions basées sur les TCC présentent une réduction de symptômes plus importante que celle obtenue avec les thérapies interpersonnelles, les thérapies de soutien et les thérapies d'orientation psychodynamique [35].

\section{Méthodologie}

\subsection{La population}

Les sujets sont issus des services de dialyse de trois hôpitaux de Liège (Belgique) et ses environs ${ }^{1}$. Les critères d'inclusion sont : avoir au moins 18 ans, être en dialyse depuis au moins trois mois, savoir lire et écrire le français, avoir un score total d'au moins 14 à l'Hospital Anxiety and Depression Scale (HADS) et enfin, accepter de participer aux 11 sessions d'intervention en signant le formulaire de consentement. Le seul critère d'exclusion était la présence d'un trouble confusionnel ou d'une démence.

\subsection{Les outils}

\subsubsection{La Hospital Anxiety and Depression Scale [36]}

Utilisée chez les patients dialysés, elle permet une évaluation de l'anxiété, de la dépression et de la détresse émotionnelle [12]. Les scores des sous-échelles d'anxiété et de dépression permettent d'identifier des niveaux faibles (0-7), moyens (8-10) et élevés (supérieurs ou égaux à 11) d'anxiété et de dépression [15]. L'échelle globale permet d'identifier des niveaux faibles (score de 0 à 12), moyens (score de 13 à 18) et élevés (score supérieur ou égal à 19) de détresse émotionnelle [37].

\subsubsection{Le Kidney Disease Quality of Life instrument Short Form [38,39]}

Celui-ci est un autoquestionnaire constitué d'une partie générique, le SF-36, et d'un module spécifique. Le SF-36 comprend 36 questions regroupées en huit dimensions correspondant chacune à un aspect différent de la santé. Le score global est ramené sur 100. Le SF-36 peut être subdivisé en une composante physique et en une composante mentale. La partie du KDQoL dévolue à l'étude spécifique de la qualité de vie chez les patients dialysés mesure les répercussions de la maladie rénale et de son traitement sur la vie quotidienne des sujets et leur degré de satisfaction. Ce module spécifique comporte 43 questions, regroupées en 11 dimensions. Chacune des questions est cotée de 0 (mauvaise qualité de vie dans le domaine investigué) à 100 (excellente qualité de vie dans le domaine investigué). Le score global, qui comprend le SF-36, est ramené sur 100. Leplège et son équipe [40] montrent que la qualité de vie de la population tout venant française au SF-36 $(n=3617)$ est de 75,04.

\subsubsection{Le questionnaire sur les inquiétudes de Penn State [41]}

Il évalue les intrusions cognitives ou les inquiétudes excessivement irréalistes, constituant les principaux symptômes du trouble de l'anxiété généralisée. De cette façon, il apporte des informations complémentaires à celles déjà apportées par le HADS. Il s'agit d'un autoquestionnaire comprenant 16 items que le sujet cote sur une échelle de 1 à 5 . Le score de cinq items doit être inversé pour la correction. Les notes obtenues aux 16 items doivent être additionnées (étendue de 16 à 80 ). Les normes, pour la version française, varient d'un score de 45,67 (écart-type =10,64) à 47,13 (écart-type $=10,14)[42]$.

${ }^{1}$ Centre Hospitalier Régional de Liège (CHR); centre hospitalier du Bois de l'Abbaye et de Hesbaye (CHBAH); centre hospitalier universitaire du Sart-Tilman (CHU). 


\subsection{La procédure}

Les interventions psychologiques individuelles ont lieu avant, après ou le lendemain de la dialyse, au choix du patient, dans un local isolé et, en face à face. Pour satisfaire aux exigences du comité d'éthique de la faculté de psychologie et des sciences de l'éducation de l'université de Liège, chaque patient reçoit une liste des services de santé mentale au terme de la thérapie.

En plus des évaluations de variables psychologiques, nous avons également rassemblé certaines données médicales relevées avant la dialyse pendant deux semaines avant et après toute intervention :

- les moyennes de prises de poids interdialytiques nous permettant de chiffrer les efforts des patients en ce qui concerne l'apport hydrique ;

- les moyennes du taux de potassium ainsi que du produit calcium phosphore nous permettant de mettre à jour les efforts du patient concernant son alimentation ainsi que sa prise de médicaments.

Les mesures d'évaluation (potassium, produit calcium-phosphore, prises de poids interdialytiques, KDQol-SF, HADS, Penn State) se déroulent selon le Tableau 1 repris ci-dessous. Le programme d'intervention, quant à lui, comporte trois modules : le module anxiété (quatre heures) le module de dépression (quatre heures) et celui de psychoéducation (trois heures). Les trois groupes de patients recrutés ont bénéficié du programme : l'ordre des modules est décrit dans le Tableau 1 et a donc été différent selon les groupes.

\subsection{Le programme d'intervention}

\subsubsection{Les objectifs}

Les objectifs principaux sont d'apporter aux patients des techniques TCC précises leur permettant d'apprendre à identifier la détresse psychologique, à mettre en place des stratégies d'interventions concrètes pour diminuer les symptômes psychologiques, et à augmenter l'adhésion au traitement et indirectement la qualité de vie.

Le module d'anxiété se focalise tout d'abord sur l'identification des réactions émotionnelles, réactions qui se traduisent habituellement par des symptômes somatiques (tachycardie, sudation) ou psychiques (pessimisme, ruminations), par des modifications comportementales (vérifications répétées et supérieures à la moyenne) et des inquiétudes excessives et incontrôlables [43].

Ensuite, le patient apprend différentes techniques d'interventions.

Le module de dépression permet au sujet de repérer s'il présente une " humeur dépressive " c'est-à-dire un état momentané de détresse, de perte d'intérêt, d'énergie et d'en train puis d'agir. Cette humeur peut survenir à la suite d'un événement stressant (ex : l'entrée dans la dialyse) lorsque le sujet ne dispose pas (ou croit ne pas disposer) des ressources (personnelles et sociales) nécessaires pour faire face.

Le module de psychoéducation est une démarche d'éducation du patient prenant en compte les causes et les conséquences de la dialyse, le contrôle des facteurs déclencheurs et les principaux aspects psychopathologiques de la dialyse, ainsi que la qualité de la relation médecin-patient. L'éducation du patient est un processus par étapes, intégré dans la démarche de soins. Elle comprend un ensemble d'activités organisées de sensibilisation, d'information, d'apprentissage et d'aide psychologique et sociale, concernant la dialyse et les traitements. Ces activités sont destinées à aider le patient (et sa famille) à comprendre la dialyse et les traitements, collaborer aux soins, prendre en charge son état de santé, et favoriser un retour aux activités normales [44,45].

\subsubsection{Les techniques d'intervention}

Dans la perspective des TCC, la prise en charge des patients s'inspire à la fois des théories de l'apprentissage et de diverses théories cognitives relatives à l'étiologie de certains troubles de la personnalité. Elle est centrée sur trois objectifs [46]. Le premier objectif est d'identifier puis de modifier les pensées erronées (exemple : croire en son incapacité à gérer ou réduire la douleur); ces distorsions cognitives seront remplacées par des pensées plus réalistes et associées à des images agréables (par diversion et imagerie). Deuxièmement, l'intervenant renforce les stratégies d'ajustement efficaces (coping), et réduit les stratégies nocives (apprendre à s'affirmer, à communiquer, à suivre des prescriptions médicales, un programme d'exercices physiques, un régime). Le troisième objectif est de réduire à la fois certaines cognitions erronées et les comportements nocifs qui y sont associés par l'utilisation systématique de plusieurs techniques : restructuration cognitive, imagerie mentale, biofeedback, relaxation.

Ces techniques, en pleine expansion, donnent en général des résultats satisfaisants [46] en améliorant le bien-être et la qualité de vie, en réduisant la douleur et en maintenant l'adhésion thérapeutique des participants. Elles semblent en outre avoir un impact favorable sur le fonctionnement du système immunitaire.

Le module anxiété s'appuie essentiellement sur trois techniques : la détection de l'anxiété, la restructuration des pensées anxieuses et la relaxation.

La détection de l'anxiété : le psychologue définit l'anxiété verbalement au patient, qui complète ensuite un inventaire des inquiétudes. Seules les inquiétudes ayant une fréquence et/ou une intensité élevées sont investiguées à l'aide de la grille SECCA ; celleci cible le stimulus (S), l'émotion (E), la cognition (C), le comportement (C) et l'anticipation (A) [47]. Pour compléter cette grille de manière exhaustive, il est demandé au patient de remplir un tableau à trois colonnes (situation - émotion - pensées automatiques) entre les deux rendez-vous.

Tableau 1

Plan expérimental selon les groupes et les moments d'intervention.

\begin{tabular}{|c|c|c|c|c|c|c|c|c|}
\hline \multirow[t]{3}{*}{ Évaluation } & \multirow[t]{3}{*}{ Groupes } & \multicolumn{3}{|c|}{ Intervention } & \multicolumn{2}{|l|}{ Intervention } & \multicolumn{2}{|l|}{ Intervention } \\
\hline & & $\mathrm{T}$ & & & & & & \\
\hline & & 0 & & 1 & & 2 & & 3 \\
\hline & 1 & & Anxiété & & Psychoéducation & & Dépression & \\
\hline & 2 & & Dépression & & Anxiété & & Psychoéducation & \\
\hline & 3 & & Psychoéducation & & Dépression & & Anxiété & \\
\hline Prise de poids & & $\mathrm{x}$ & & & & & & $\mathrm{x}$ \\
\hline Potassium & & $\mathrm{x}$ & & & & & & $\mathrm{x}$ \\
\hline Produit calcium-phosphore & & $\mathrm{x}$ & & & & & & $\mathrm{x}$ \\
\hline KDQoL-SF & & $\mathrm{x}$ & & & & & & $\mathrm{x}$ \\
\hline Penn State & & $\mathrm{x}$ & & $\mathrm{x}$ & & $\mathrm{x}$ & & $\mathrm{x}$ \\
\hline HADS & & $\mathrm{x}$ & & $\mathrm{x}$ & & $\mathrm{x}$ & & $\mathrm{x}$ \\
\hline
\end{tabular}


La restructuration des pensées anxieuses se travaille à partir du tableau complété entre les deux rendez-vous. Le psychologue détecte, avec le patient, ses pensées négatives ou irrationnelles et les remplace par des pensées plus positives et plus constructives. L'exercice se poursuit avec un tableau à quatre colonnes (situation - émotion - pensées automatiques - pensées alternatives) que le patient remplit seul en présence d'anxiété.

La relaxation est enseignée au patient dans le but de diminuer son anxiété ainsi que les tensions musculaires qui l'accompagnent. La séance proposée au patient dure 25 minutes et s'inspire de la technique de Jacobson [48,49], c'est-à-dire que la relaxation se fait par des contractions suivies de décontractions de divers groupes musculaires. La contraction est réalisée en même temps qu'une inspiration profonde et elle est suivie d'une courte apnée. Ensuite, le patient décontracte le groupe musculaire en même temps qu'il expire lentement et qu'il énonce intérieurement un mot clé évoquant la détente (relax). Pour prolonger les effets bénéfiques de cette séance, un $C D$ contenant exactement la même séance de relaxation est donné au patient afin qu'il puisse la pratiquer quand il le souhaite.

Le module dépression s'appuie essentiellement sur quatre techniques : l'identification de la dépression, l'entretien motivationnel, l'activation comportementale ou implémentation d'action et la restructuration cognitive.

L'identification de la dépression : une série d'informations est donnée au patient dialysé sur le moral, les symptômes de dépression, ainsi que les paradigmes cognitif et comportemental. Le premier paradigme implique que les pensées sont des interprétations de la réalité mais pas la réalité elle-même. La réponse émotionnelle à un événement est déterminée par la signification personnelle qu'on lui accorde. Le second mène à une rétroaction entre le manque de motivation, qui diminue le niveau d'activité, et le niveau d'activité, qui diminue la motivation au travers de la culpabilité et du découragement [47]. Pendant cette première séance, le psychologue effectue une évaluation de l'emploi du temps du patient. Qu'a-t-il laissé de côté à l'entrée en dialyse ? Existe-t-il une activité qu'il souhaite mettre en place depuis longtemps ? Un tableau d'enregistrement des activités quotidiennes est également donné au patient afin de poursuivre l'évaluation entre les deux rendez-vous.

L'entretien motivationnel poursuit deux objectifs : aider les sujets à se motiver eux-mêmes et à rester motivés pour changer de comportement, et s'appuyer sur la résistance du sujet pour développer sa collaboration active. Le thérapeute est empathique ; il cherche à développer les contradictions entre le comportement actuel et les buts à long terme, ce qui est un puissant moteur de changement, permettant une bonne prise de conscience des conséquences, donc de la réalité du sujet. Le thérapeute encourage l'estime de soi de façon à ce que le sujet croie en sa capacité de changer, donc développe sa motivation [47].

L'implémentation d'action ou activation comportementale consiste à augmenter les activités d'un patient afin qu'il puisse être en contact avec des contingences environnementales de type renforcement qui lui permettront d'améliorer sa situation en termes de pensée, humeur et qualité de vie [50-52].

Elle est sous-tendue par trois principes clés :

- la motivation suit l'action. Il est important d'expliquer au patient que c'est en mettant en œuvre une activité qu'il va en tirer satisfaction, ce qui entraînera une motivation à renouveler l'opération :

- l'action est simple et concrète. Les objectifs fixés sont toujours proximaux, c'est-à-dire qu'un minimum de temps et d'efforts doivent être réalisés pour atteindre un premier objectif, puis un second jusqu'à atteindre l'objectif principal ;

- l'action est agréable et/ou gratifiante. Un « feedback » est donné au patient par le thérapeute à chaque séance sur les tâches réalisées hors sessions. Ce " feedback » est constitué majoritairement de renforcements positifs et de commentaires/suggestions sur les améliorations possibles. Ce " feedback » constitue déjà un aspect non négligeable de la thérapie puisqu'il améliore le sentiment d'efficacité personnelle, il suscite davantage d'intérêts et améliore dès lors la performance même du patient. Il est d'ailleurs indispensable au sein même des implémentations d'actions puisqu'il encourage le patient à avancer dans l'atteinte de ses différents objectifs [53]. Signalons que certaines situations peuvent nécessiter une résolution de problème au cours de laquelle, toutes les solutions possibles seront investiguées (avantages - inconvénients de chaque solution à court et à long termes) avant de choisir la solution optimale.

La restructuration cognitive consiste à identifier les pensées négatives via un tableau à trois colonnes dont nous avons déjà parlé. À partir de ces différentes observations, le thérapeute apprend au patient à mettre en question ses pensées automatiques. Après avoir examiné l'évidence qui supporte la pensée automatique et l'évidence contradictoire à cette même pensée, le thérapeute peut poursuivre le travail en posant quelques questions clés : " que pourrait-on penser d'autres dans cette même situation ? " J'imagine une personne que je connais bien et qui serait dans la même situation, que penserait-elle ? " Il s'agit de la recherche systématique d'autres points de vue possibles (hypothèses alternatives). Comme tâche à domicile, le patient poursuivra cette réflexion à l'aide du tableau à quatre colonnes à compléter en présence d'émotions négatives.

Le module de psychoéducation est construit à partir de la connaissance du patient concernant l'insuffisance rénale, le traitement de suppléance, les médicaments ainsi que l'alimentation. Des précisions et des conseils sont apportés sur les limitations nécessaires en potassium, en phosphore et en liquides ainsi que sur l'hygiène de vie en général. Deux autres variables sont particulièrement discutées : le sommeil et l'alimentation. En effet, le sommeil est régulièrement perturbé chez les patients dialysés [54]. C'est pourquoi, une évaluation de la qualité du sommeil par un relevé précis des heures d'endormissement, de réveils nocturnes, de siestes et d'éveils matinaux est réalisée.

Ensuite, toute une série de conseils visant à améliorer l'hygiène du sommeil est donnée. Le même procédé a été utilisé pour le statut nutritionnel des patients.

\subsection{Les statistiques}

Toutes les analyses statistiques ont été réalisées avec la version 8 de Statistica.

Le critère d'égalité des variances est respecté pour toutes les variables étudiées tandis que la distribution des variables n'est pas respectée pour toutes les variables. Néanmoins, la normalité des données n'est pas un critère absolument essentiel pour les analyses de variance. Dès lors, nous avons eu recours à des statistiques paramétriques.

Les comparaisons entre les résultats obtenus par les patients avant et après chacun des modules ont été réalisées avec des analyses de variance en mesures répétées.

\section{Résultats}

\subsection{Statistiques élémentaires}

Les données sociodémographiques portent sur trois groupes de patients : le premier groupe est constitué de 19 patients dont sept femmes $(59,8$ ans $\pm 17,4)$. Le deuxième a permis de recruter 16 patients dont dix femmes $(67,6$ ans $\pm 14,1)$. Le troisième groupe se compose de 12 patients dont quatre femmes $(61,8$ ans $\pm 17,7)$. Aucune différence statistique significative n'est observée entre les trois groupes tant au niveau de l'âge que de la répartition du genre. 
Pour l'ensemble des trois groupes, 18 sont dialysés au CHR, 16 au CHU, 13 au CHBAH. Aucune différence statistique significative n'est observée entre les trois centres, et ce pour aucune des variables étudiées.

Au début des interventions (T0), 47 patients hémodialysés ont accepté de participer à l'étude. À la fin des interventions (T3), les effectifs se répartissent comme suit : pour le premier groupe, 14/19 $(73,68 \%)$ patients ; pour le deuxième, $12 / 16$ (75\%) et enfin pour le troisième, neuf sur 12 (75\%). Les différents motifs expliquant les abandons sont : décès $(n=1)$, dégradation de la santé $(n=4)$, emploi du temps $(n=5)$, confusion du patient empêchant les interventions $(n=1)$ et souhait de se retirer de l'étude $(n=1)$.

Le taux moyen de participation aux séances est de $9 / 11$ pour les trois groupes (> $80 \%$ ).

\subsection{Corrélations}

On observe une corrélation importante entre les prises de poids interdialytiques et le produit calcium-phosphore $(r=0,48$; $p=0,001)$. Une tendance en ce sens existe entre les prises de poids et le taux de potassium $(r=0,24 ; p=0,10)$. Ces résultats indiquent qu'un patient, veillant à limiter sa prise de poids entre deux dialyses, sera également plus vigilant par rapport à ses apports de phosphore, de potassium et à la médication contrôlant ces deux variables.

Les questionnaires psychologiques corrèlent entre eux comme attendu, c'est-à-dire que des corrélations positives modérées existent entre anxiété et inquiétudes $(r=0,41 ; p=0,004)$, entre anxiété et dépression $(r=0,31 ; p=0,04)$ ainsi qu'une corrélation négative modérée entre dépression et qualité de vie $(r=-0,35$; $p=0,02$ ). Ces corrélations nous indiquent que chaque échelle évalue un thème bien spécifique et permet d'aborder la détresse émotionnelle dans sa multifactorialité.

\subsection{Impact des interventions}

\subsubsection{Statistiques descriptives avant l'intervention (TO)}

Dans le Tableau 2, les résultats au T0 (ligne de base) sont présentés pour l'ensemble des sujets et pour chacun des groupes séparément.

Pour l'ensemble des sujets $(n=47)$, le score de détresse émotionnelle (HADS total) est de plus de 21 ce qui correspond à un score élevé. Par ailleurs, les scores obtenus par le groupe 3 montrent une détresse émotionnelle (inquiétudes, anxiété et dépression) plus sévère que les deux autres groupes. Ces différentes observations doivent être nuancées compte tenu du critère de sélection stipulant que seuls les patients présentant un score supérieur à 14 à l'HADS peuvent bénéficier des interventions.

Toujours pour l'ensemble des sujets, le score total de qualité de vie obtenu au SF-36 est de 36,85, indice d'une mauvaise qualité de vie, alors que, pour la population française générale, il est de 75,05. Les résultats des patients dialysés $(n=47)$ sont comparables à ceux retrouvés chez 133 patients dialysés en 2008 [15] ; ils sont aussi significativement inférieurs à ceux retrouvés dans d'autres maladies chroniques comme le cancer [15].

Enfin, les données médicales au T0 sont semblables à celles retrouvées dans une population de patients dialysés tout venants. Ainsi, et au vu de la présence des patients à chaque dialyse (sauf exceptions), nous étions déjà au T0 face à un profil d'adhésion tout à fait intéressant.

\subsubsection{Comparaison des résultats par groupe au TO}

Les analyses de variance montrent premièrement que le groupe 2 est significativement moins inquiet et moins anxieux que les deux autres groupes ; deuxièmement, il existe une tendance pour le groupe 2 à présenter un score de dépression moins élevé que les deux autres groupes.

L'analyse de variance montre également que le groupe 2 présente un produit calcium phosphore plus bas que les deux autres groupes (Tableau 2).

\subsubsection{Comparaison des résultats (T0-T3), tous groupes confondus}

Des analyses de variance (Anova) sont effectuées dans le but de déterminer si les résultats obtenus par les participants varient au fil du suivi (Tableau 3) : T0 (avant toute intervention), T1 (après les quatre premières séances) et T3 (à la fin des interventions).

Sur le plan médical, les prises de poids et le taux de potassium n'évoluent pas entre T0 et T3 tandis que le produit calciumphosphore diminue significativement (Tableau 3). Plus précisément, quand on compare les personnes ayant bénéficié du module psychoéducation ou pas, seules les personnes ayant bénéficié du module de psychoéducation $(n=39 / 47)$ montrent une diminution significative de cette variable $(F=5,969$; $p=0,019$ ). Sur le plan psychologique, à l'exception de la composante physique (sous-score du SF-36) qui ne s'améliore pas de manière significative, toutes les autres variables indiquent une nette amélioration.

Au bout de quatre interventions (T0-T1), on peut déjà constater que les scores d'anxiété et de dépression diminuent significativement. Ce n'est pas le cas des inquiétudes (Tableau 3).

Tableau 2

Moyennes et écart-types des données psychologiques au temps 0 et comparaisons de ces données entre les trois groupes par des analyses de variance.

\begin{tabular}{|c|c|c|c|c|c|c|c|c|c|}
\hline \multirow[t]{2}{*}{ Variables } & \multicolumn{2}{|c|}{ Total $(n=47)$} & \multicolumn{2}{|c|}{ Groupe $1(n=19)$} & \multicolumn{2}{|c|}{ Groupe $2(n=16)$} & \multicolumn{2}{|c|}{ Groupe $3(n=12)$} & \multirow[t]{2}{*}{$\mathrm{F}$} \\
\hline & Moyenne & S.D. & Moyenne & S.D. & Moyenne & S.D. & Moyenne & S.D. & \\
\hline Prise de poids & 2,10 & 1,09 & 2,10 & 1,18 & 2,12 & 1,00 & 2,04 & 1,14 & 0,02 \\
\hline Taux potassium $^{\mathrm{a}}$ & 4,78 & 0,76 & 4,66 & 0,57 & 4,79 & 0,65 & 4,98 & 1,11 & 0,65 \\
\hline Produit calcium-phosphore ${ }^{\mathrm{b}}$ & 53,10 & 15,77 & 57,66 & 16,17 & 45,68 & 8,79 & 55,79 & 19,56 & $2,97^{*}$ \\
\hline Penn State & 48 & 9,39 & 46 & 8,27 & 44,69 & 9,7 & 55,58 & 6,56 & $6,65^{* *}$ \\
\hline HADS total & 21,13 & 5,84 & 22,89 & 4,99 & 17,06 & 3,99 & 23,75 & 6,59 & $7,67^{* *}$ \\
\hline HAD-Anxiété & 11,17 & 3,71 & 12 & 3,28 & 8,56 & 3,03 & 13,33 & 3,37 & $8,58^{* *}$ \\
\hline HAD-Dépression & 9,96 & 3,51 & 10,89 & 3,36 & 8,5 & 2,76 & 10,42 & 4,23 & 2,27 \\
\hline KDQoL-SF Total & 44,54 & 11,63 & 42,02 & 13,34 & 46,04 & 11,14 & 46,52 & 9,29 & 0,75 \\
\hline KDQoL spécifique & 50,58 & 11,31 & 47,34 & 13,36 & 53,58 & 10,41 & 51,71 & 7,91 & 1,43 \\
\hline SF-36 Total & 36,85 & 15,32 & 35,28 & 16,16 & 36,47 & 14,95 & 39,83 & 15,3 & 0,32 \\
\hline SF-36-mentale & 36,70 & 15,78 & 34,71 & 15,52 & 39,79 & 17,46 & 35,71 & 14,48 & 0,47 \\
\hline SF-36-physique & 36,98 & 17,88 & 35,67 & 19,62 & 53,58 & 10,41 & 42,58 & 17,33 & 0,81 \\
\hline
\end{tabular}

${ }^{*} p \leq 0,10 ;{ }^{* *} p \leq 0,01$.

a Exprimé en micromol par litre.

b Taux de calcium $\times$ taux de phosphore. 
Tableau 3

Statistiques F obtenues par des Anova entre T0 et T3 et entre T0 et T1.

\begin{tabular}{|c|c|c|c|c|c|c|}
\hline & \multirow{2}{*}{$\begin{array}{l}\text { T0 } \\
n=47\end{array}$} & \multirow{2}{*}{$\begin{array}{l}\text { T3 } \\
n=46\end{array}$} & \multirow[t]{2}{*}{$\mathrm{F}$} & \multirow{2}{*}{$\frac{\text { T0 }}{n=47}$} & \multirow{2}{*}{$\begin{array}{l}\mathrm{T} 1 \\
n=37\end{array}$} & \multirow[t]{2}{*}{$\mathrm{F}$} \\
\hline & & & & & & \\
\hline Prise de poids & $\begin{array}{l}2,10 \\
( \pm 1,09)\end{array}$ & $\begin{array}{l}2,12 \\
( \pm 1,14)\end{array}$ & 0,007 & $\begin{array}{l}2,10 \\
( \pm 1,09)\end{array}$ & & \\
\hline Taux potassium* & $\begin{array}{l}4,78 \\
( \pm 0,76)\end{array}$ & $\begin{array}{l}4,65 \\
( \pm 0,67)\end{array}$ & 2,559 & $\begin{array}{l}4,78 \\
( \pm 0,76)\end{array}$ & & \\
\hline Produit calcium-phosphore ${ }^{* *}$ & $\begin{array}{l}53,10 \\
( \pm 15,77)\end{array}$ & $\begin{array}{l}47,24 \\
( \pm 17,29)\end{array}$ & $5,906 *$ & $\begin{array}{l}53,10 \\
( \pm 15,77)\end{array}$ & & \\
\hline & & $n=35$ & & & & \\
\hline Penn State & $\begin{array}{l}48 \\
( \pm 9,39)\end{array}$ & $\begin{array}{l}43,8 \\
( \pm 12,06)\end{array}$ & $4,966^{*}$ & $\begin{array}{l}48 \\
( \pm 9,39)\end{array}$ & $\begin{array}{l}45,89 \\
( \pm 10,28)\end{array}$ & 0,895 \\
\hline HAD-anxiété & $\begin{array}{l}11,17 \\
( \pm 3,71)\end{array}$ & $\begin{array}{l}8,6 \\
( \pm 4,78)\end{array}$ & $15,4399^{* *}$ & $\begin{array}{l}11,17 \\
( \pm 3,71)\end{array}$ & $\begin{array}{l}9,41 \\
( \pm 3,56)\end{array}$ & $6,774^{*}$ \\
\hline HAD-dépression & $\begin{array}{l}9,96 \\
( \pm 3,51)\end{array}$ & $\begin{array}{l}8,09 \\
( \pm 4,42)\end{array}$ & $7,909^{* *}$ & $\begin{array}{l}9,96 \\
( \pm 3,51)\end{array}$ & $\begin{array}{l}8,16 \\
( \pm 3,40)\end{array}$ & 9,771 $1^{* *}$ \\
\hline KDQoL-SF Total & $\begin{array}{l}44,54 \\
( \pm 11,63)\end{array}$ & $\begin{array}{l}49,19 \\
( \pm 16,55)\end{array}$ & $17,186^{* *}$ & $\begin{array}{l}44,54 \\
( \pm 11,63)\end{array}$ & & \\
\hline KDQoL spécifique & $\begin{array}{l}50,58 \\
( \pm 11,31)\end{array}$ & $\begin{array}{l}54,72 \\
( \pm 15,76)\end{array}$ & $13,65^{* *}$ & $\begin{array}{l}50,58 \\
( \pm 11,31)\end{array}$ & & \\
\hline SF-36 Total & $\begin{array}{l}36,85 \\
( \pm 15,32)\end{array}$ & $\begin{array}{l}42,32 \\
( \pm 20,06)\end{array}$ & $11,152^{* *}$ & $\begin{array}{l}36,85 \\
( \pm 15,32)\end{array}$ & & \\
\hline SF-36 physique & $\begin{array}{l}36,98 \\
( \pm 17,87)\end{array}$ & $\begin{array}{l}40,24 \\
( \pm 20,87)\end{array}$ & 3,396 & $\begin{array}{l}36,98 \\
( \pm 17,87)\end{array}$ & & \\
\hline SF-36 mentale & $\begin{array}{l}36,70 \\
( \pm 15,78)\end{array}$ & $\begin{array}{l}45,45 \\
( \pm 22,43)\end{array}$ & $18,89^{* *}$ & $\begin{array}{l}36,70 \\
( \pm 15,78)\end{array}$ & & \\
\hline
\end{tabular}

\section{Discussion}

L'objectif de cette étude est de tester l'intérêt et la faisabilité d'une approche psychologique individuelle longitudinale dans le but de diminuer la détresse émotionnelle (anxiété, inquiétudes et dépression) des patients IRT. De manière indirecte, nous nous attendions à une amélioration de la qualité de vie et de la santé physique : diminution de la prise de poids interdialytique, du taux de potassium et du produit calcium-phosphore. Pour ce faire, nous avons suivi l'évolution de 47 patients pendant trois mois.

Un premier résultat indique que le taux de participation au programme d'intervention psychologique individuelle est supérieur à $80 \%$ (au moins neuf séances sur 11). Ce taux de participation satisfaisant permet de conclure à l'applicabilité du programme TCC en dialyse malgré des conditions complexes inhérentes à ce type de suivi médical : lourdeur des traitements, rythme des séances avec horaires parfois contraignants et fatigue du patient.

Les données médicales, quant à elles, mettent en évidence la stabilité des taux de potassium et des prises de poids interdialytiques tout au long de la prise en charge psychologique. Nous pouvons dire que ce résultat est intéressant étant donné le profil d'adhésion déjà très satisfaisant au T0 des patients des trois groupes. En revanche, le produit calcium-phosphore diminue significativement en fin d'intervention uniquement pour les patients ayant bénéficié du module psychoéducation. Il semble important de rappeler que la régulation du comportement alimentaire (dans ce cas particulier du taux de potassium, des prises de poids entre deux dialyses...) est un mécanisme complexe. Premièrement, le sujet doit mettre en œuvre à la fois des processus comportementaux, émotionnels et cognitifs pour acquérir une alimentation saine et équilibrée. Il s'agit donc pour le patient de se réconcilier avec la nourriture. Deuxièmement, maintenir cette alimentation équilibrée nécessite une vigilance permanente vis-à-vis de la nourriture afin de ne pas retourner à des habitudes alimentaires antérieures peu saines. Cette façon de procéder fait suite au constat pouvant être fait tant en diabétologie qu'en néphrologie : le régime (alimentaire, hydrique) impose certaines restrictions parfois difficiles à accepter qui viennent compliquer encore un peu plus l'aspect durable du régime des patients chroniques [55]. C'est pourquoi, notre module de psychoéducation en TCC avait pour objectifs, d'une part, d'éviter au maximum les restrictions et, d'autre part, de mettre en place des compromis durables. En fait, notre module a favorisé une prise de conscience de toutes les ambiguités liées au comportement alimentaire pour ensuite effectuer un travail de motivation constructif. Nous pensons également que pour un certain nombre de patients, la diminution de leur détresse émotionnelle a pu leur permettre de se concentrer sur la prise en charge de leur santé physique.

Ainsi, d'autres études menées en dialyse montrent des tentatives de diminution du phosphore par des programmes de psychoéducation proposés aux patients dialysés, sans succès équivalent [56,57]. En revanche, en prédialyse, la psychoéducation, comme dans notre étude, se montre efficace avec des interventions portant directement sur le statut nutritionnel des patients. Ainsi, une information sur le régime à suivre ainsi qu'un soutien dans la poursuite des objectifs fixés donne de bons résultats, tant au niveau de la santé physique qu'au niveau de la qualité de vie [58]. Des interventions plus globales comprenant des informations, de l'éducation, des encouragements, de l'« empowerment ${ }^{2}$ " aboutissent à une réduction de $41 \%$ de risque de décès dans les 90 premiers jours suivant l'entrée en dialyse [59].

Ensuite, les données psychologiques entre T0 et T3 mettent en évidence de manière significative une diminution des inquiétudes,

\footnotetext{
${ }^{2}$ Capacité d'une personne de comprendre ce qui peut déterminer sa qualité de vie, et d'agir en prenant des décisions et en exerçant un contrôle sur sa vie personnelle en vue de maintenir ou d'améliorer sa qualité de vie.
} 
de la détresse émotionnelle (anxiété et dépression), et une amélioration généralisée de la qualité de vie à la fin des interventions. En ce qui concerne la dépression et la qualité de vie, nos résultats vont dans le même sens que les études réalisées par Tsay et al., [25], Lii et al. [26], et Duarte et al. [60]. Dans la première étude, réalisée en 2005 , les auteurs ont mis au point et testé un programme d'adaptation à la maladie rénale visant à diminuer le niveau de dépression des patients et à améliorer leur qualité de vie [25]. Leur programme est très similaire au nôtre puisqu'il s'inspire des TCC. Les évaluations réalisées trois mois après avoir délivré le programme montrent des différences significatives entre le groupe expérimental $(n=33)$ et le groupe témoin $(n=33)$. Ainsi, les patients ayant suivi le programme se considéraient comme moins stressés, présentaient un niveau de dépression moins élevé ainsi qu'une meilleure qualité de vie que les patients issus du groupe témoin. Dans la deuxième étude, réalisée en 2006, les auteurs ont effectué le même type de recherche en utilisant des interventions psychosociales de groupe (de nouveau similaires aux nôtres puisqu'elles s'inspirent des TCC) [26]. Après les interventions, les résultats indiquent un niveau de dépression significativement plus faible au sein du groupe expérimental $(n=20)$ par rapport aux sujets du groupe témoin $(n=20)$. Une troisième étude réalisée en 2009 montre qu'en dialyse, le développement de stratégies de " coping » [46], la restructuration cognitive, la gestion des émotions, la résolution de conflit, l'implémentation d'actions (afin d'augmenter les activités) ainsi que le développement d'habiletés sociales ont conduit à une diminution significative du score de dépression au sein du groupe expérimental $(n=41)[60]$.

Toutes les études citées précédemment sont réalisées avec des échantillons comparables aux nôtres ainsi qu'avec des interventions TCC. En revanche, notre étude va plus loin dans la prise en charge de la détresse émotionnelle en travaillant les notions d'anxiété et d'inquiétudes, qui dans le cas de la dialyse, peuvent être invalidantes. En effet, les TCC permettent de diminuer la fréquence et l'intensité des inquiétudes chroniques ainsi que de l'anxiété. Elles sont plus efficaces qu'un traitement psychologique non directif principalement composé d'écoute active, d'intervention empathique et d'éléments de support [61]. Ainsi, notre programme de 11 séances donne des résultats intéressants mais que ce serait-il passé si le soutien s'était limité aux quatre premières séances ? Tout d'abord, la diminution des inquiétudes est très progressive, on ne note pas encore de diminution significative entre T0 et T1. En effet, si l'anxiété est une émotion, les inquiétudes, elles, sont des phénomènes cognitifs bien ancrés, liés à l'intolérance et à l'incertitude [62,63]. Notre expérience clinique nous amène à penser qu'il est indispensable de diminuer préalablement les taux d'anxiété et de dépression pour laisser émerger petit à petit les inquiétudes normales. Le patient a besoin de plus de temps pour faire la part entre ses ruminations et la valeur adaptative de ses inquiétudes. Plus précisément, les deux premiers modules ont pu dégager les symptômes anxieux et dépressifs, faisant place à des inquiétudes plus concrètes et pouvant être plus facilement résolues au cours du troisième module.

Si nos résultats sont très prometteurs, nous constatons néanmoins deux limites au sein de notre étude : aucun groupe témoin n'a été intégré dans le protocole scientifique ; les rôles de thérapeute et d'évaluateur étaient tenus par une même personne. Ces deux limites sont liées aux conditions de mise en place du protocole : des restrictions budgétaires ne nous permettaient pas de faire appel à un second intervenant, ce qui aurait pu permettre la séparation des rôles thérapeute-évaluateur. Des restrictions temporelles nous ont empêché de constituer un groupe témoin. À l'avenir, si nous pouvons mettre en place un nouveau protocole de recherche, ces deux limites seront impérativement corrigées.
En revanche, le point fort de notre étude est la standardisation des interventions.

Au vu de tous ces résultats et étant donné l'efficacité de notre programme, alors même que les patients étaient déjà installés dans la maladie et dans une certaine détresse psychologique, nous nous demandons à quel point ce même programme, légèrement modifié, pourrait être utile à l'entrée en dialyse. Il s'agirait alors de prévention secondaire. L'essentiel serait de fournir à chacun des patients, idéalement déjà avant leur entrée en dialyse, des informations et des outils, leur permettant de mieux gérer l'insuffisance rénale et son traitement. Nous nous placerions alors dans une perspective de psychologie de la santé où la maladie est déterminée par une combinaison de facteurs biologiques, psychologiques (ex. : des comportements, des croyances) et sociaux (ex. : la précarité). Nos objectifs pourraient être, entre autres, la promotion de comportements sains pour la santé, la diminution du stress afin de réduire les risques cardiovasculaires secondaires. La prise en charge des conséquences psychologiques de l'annonce de la maladie et de son traitement pourrait aussi avoir un impact sur la longévité, etc. [45].

\section{Conclusion}

Notre étude, la première du genre dans les pays francophones, a démontré l'intérêt d'un soutien psychologique TCC au patient dialysé. L'état physique des patients est aussi amélioré comme en témoigne la diminution du produit calcium-phosphore. Notre programme permet de diminuer les symptômes négatifs et aussi d'augmenter les affects positifs permettant aux patients de présenter des cognitions et des comportements qui vont dans le sens d'une meilleure qualité de vie. De plus, on sait que l'affectivité positive est corrélée négativement à la dépression tandis que l'affectivité négative est corrélée positivement à la dépression [15]. Ce programme, utilisé en prévention, pourrait notamment permettre de retarder, de contrecarrer l'apparition d'une dépression, délétère pour la qualité de vie du patient IRT.

\section{Conflit d'intérêt}

Aucun.

\section{Références}

[1] Groupement des néphrologues francophones de Belgique, de nederlandstalige belgische vereniging voor nefrologie. Rapport commun du GNFB et du NBVN pour le Collège de néphrologie. Collège de néphrologie 2007. Disponible sur : URL : http://www.gnfb.be/rapportreunion/Rapport_commun_2007.pdf.

[2] Cleary J, Drennan J. Quality of life of patients on hemodialysis for end-stage renal disease. J Adv Nurs 2004;51(6):577-86.

[3] Guzman SJ, Nicassio PM. The contribution of negative and positive illness schemas to depression in patients with end-stage renal disease. J Behav Med 2003;26(6):517-34.

[4] Finkelstein FO, Watnik S, Finkelstein SH, Wuerth D. The treatment of depression in patients maintained on dialysis. J Psychosom Res 2002;53(4):957-60.

[5] Takaki J, Nishi T, Shimoyama H, Inada T, Matsuyama N, Kumano H. Possible interactive effects of demographic factors and stress coping mechanisms on depression and anxiety in maintenance hemodialysis patients. J Psychosom Res 2005;58(3):217-23.

[6] Antoine V, Edy T, Souid M, Barthélémy F, Saint-Jean O. Le point sur : vieillissement, entrée en dialyse, entrée en dépendance : répercussion sur le psychisme du patient dialysé très âgé. Nephrologie 2004;25(3):83-8.

[7] Cvengros JA, Christensen AJ, Lawton WJ. Health locus of control and depression in chronic kidney disease: a dynamic perspective. J Health Psychol 2005;10(5):677-86.

[8] Yamamoto Y, Hayashino Y, Akiba T, Akizawa, Asano Y, Saito A, et al. Depressive symptoms predict the subsequent risk of bodily pain in dialysis patients: Japan dialysis outcomes and practice patterns study. Pain Med 2009; 10(5):883-9.

[9] Chilcot J, Wellsted D, Farrington K. Screening for depression while patients dialyse: an evaluation. Nephrol Dial Transplant 2008;23:2653-9.

[10] Yamamoto Y, Hayashino Y, Yamazaki S, Akiba T, Akizawa T, Asano y. et al. Depressive symptoms predict the future risk of severe pruritus in haemodia- 
lysis patients: Japan dialysis outcomes and practice patterns study. Br J Dermatol 2009;161:384-9.

[11] Paez AE, Jofre MJ, Azpiroz C, Angel De Bortoli M. Ansiedad y depression en pacientes con insuficiencia renal cronica en tratamiento de dialysis. Univ Psychol 2009;8(1):117-24.

[12] Untas A, Aguirrezabal M, Chauveau P, Leguen E, Combe C, Rascle N. Anxiété et dépression en hémodialyse : validation de l'Hospital Anxiety and Depression Scale (HADS). Nephrol Ther 2009;5:193-200.

[13] Cukor D, Coplan J, Brown C, Friedman S, Newville H, Safier M. Anxiety disorders in adults treated by hemodialysis: a single center study. Am J Kid Dis 2008;52(1):128-36.

[14] Devémy N, Antoine P, Grenier JL, Beaune D. Étude exploratoire des stresseurs relatifs aux maladies somatiques chroniques : identification des dimensions et analyse des liens avec la qualité de vie. Eur Rev Appl Psychol 2006;56(2):83-93.

[15] Étienne AM, Rocha C. Rapport interne sur les besoins psychosociaux des patients dialysés (manuscrit non publié). Université de Liège: Psychologie de la Santé; 2008.

[16] Cummings KM, Becker MH, Kirscht JP, Levin NW. Intervention strategies to improve compliance with medical regimens by ambulatory hemodialysis patients. J Behav Med 1981;4(1):111-27.

[17] Levin A, Lewis M, Mortiboy P, Faber S, Hare I, Porter EC, et al. Multidisciplinary predialysis programs: quantification and limitations of their impact on patient outcomes in two Canadian settings. Am J Kidney Dis 1997;29(4):533-40.

[18] Sagawa M, Oka M, Chaboyer W. The utility of cognitive behavioural therapy on chronic haemodialysis patients' fluid intake: a preliminary examination. Int J Nurs Stud 2003;40(4):367-73.

[19] Hörl WH. A need for an individualized approach to end-stage renal disease patients. Nephrol Dial Transplant 2002;17(6):17-21.

[20] Devins GM, Mendelssohn DC, Barré PE, Binik YM. Predialysis psychoeducational intervention and coping styles influence time to dialysis in chronic kidney disease. Am J Kidney Dis 2003;42(4):693-703.

[21] Goovaerts T, Jadoul M, Goffin E. Influence of a pre-dialysis education programme on the mode of renal replacement therapy. Nephrol Dial Transplant 2005;20:1842-7.

[22] Klang B, Björvell H, Clyne N. Predialysis education helps patients choose dialysis modality and increases disease specific knowledge. J Adv Nurs 1999;29(4):869-76.

[23] Kirschenbaum DS, Sherman J, Penrod JD. Promoting self-directed hemodialysis: measurement and cognitive-behavioral intervention. Health Psychol 1987;6(5):373-85.

[24] Kouidi E. Exercise training in dialysis patients: why, when and how? Artif Organs 2002;26(12):1009-13.

[25] Tsay SL, Lee YC, Lee YC. Effects of an adaptation training programme for patients with end-stage renal disease. J Adv Nurs 2005;50(1):39-46.

[26] Lii YC, Tsay SL, Wang TJ. Group intervention to improve quality of life in haemodialysis patients. J Clin Nurs 2006;16(11):268-75.

[27] Fontaine O, Kulbertus H, Étienne AM. Stress et cardiologie. Paris: Masson; 1996

[28] Williams RB, Littman AB. Psychosocial factors: role in cardiac risk and treatment strategies. Cardiol Clin 1996;14:97-104.

[29] Bennet P, Caroll D. Cognitive-behavioural interventions in cardiac rehabilitation. J Psychosom Res 1994;38:169-82.

[30] Blumenthal JA, Sherwood A, Babyak MA, Watkins LL, Waugh R, et al. Effects of exercise and stress management training on markers of cardiovascular risk in patients with ischemic heart disease. JAMA 2005;293(13):1626-34.

[31] Blumenthal JA, Wei J. Psychobehavioral treatment in cardiac rehabilitation. Cardiol Clin 1993;11:323-31

[32] Edelman S, Lemon J, Kidman A. Group cognitive behavior therapy for breast cancer patients: a qualitative evaluation. Psychol Health Med 2005;10(2): $139-44$.

[33] Edelman S, Kidman AD. Description of a group cognitive behaviour therapy program with cancer patients. Psycho Oncol 1999;8:306-14.

[34] Serfaty MA, Hawoth D, Blanchard M, Buszewicz M, Murad S, King M. Clinical effectiveness of individual cognitive behavioral therapy for depressed older people in primary care. Arch Gen Psychiatry 2009;66(12):1332-40.

[35] Churchill R, Hunot V, Cornet R, Knapp M, McGuire H, Tylee A, et al. A systematic review of controlled trials of the effectiveness and cost-effectiveness of brief psychological treatments for depression. Health Technol Assess 2001;5(35): $1-173$.

[36] Zigmond AS, Snaith RP. The Hospital Anxiety and Depression Scale. Acta Psychiatr Scand 1983;67(6):361-70.
[37] Razavi D, Delvaux N, Farvacques C, Robaye E. Screening for adjustment disorders and major depressive disorders in cancer in-patients. Br J Psychiatry 1990;156:79-83.

[38] Hays RD, Kallich JD, Mapes DL, Coons SJ, Amin L, Carter WB. Kidney disease quality of life short form, version 1.3: a manual for use and scoring. Santa Monica 1995; Rand.p. 7994.

[39] Boini S, Leplège A, Loos Ayav C, Français P, Ecosse E, Briançon S. Mesure de la qualité de vie dans l'insuffisance rénale chronique terminale : adaptation transculturelle et validation du questionnaire spécifique Kidney Disease Quality of Life. Nephrol Ther 2007;3(6):372-83.

[40] Leplège $A$, Ecosse $E$, Pouchot J, Coste J, Perneger T. Le questionnaire MOS SF36 : manuel de l'utilisateur et guide d'interprétation des scores. Paris: Estem; 2001.

[41] Meyer TJ, Miller ML, Metzger RL, Borkovec TD. Development and validation of the Penn State worry questionnaire. Behav Res Ther 1990;28:487-95.

[42] Ladouceur R, Freeston MH, Dumond J. The Penn State worry questionnaire: psychometric prosperties of a French translation. Paper presented at the annual convention of the Canadian psychological association, Quebec City Canada; 1992.

[43] Guelfi JD. L'anxiété généralisée : actualité et devenir. France: Flammarion; 2003.

[44] Deccache A, La Vendhomme E. Information et éducation du patient : des fondements aux méthodes. Bruxelles: De Boeck; 1989.

[45] Bury JA. Éducation pour la santé : concepts, enjeux, planifications. Bruxelles: De Boeck; 1988.

[46] Bruchon-Schweitzer M. Psychologie de la santé : modèles, concepts et méthodes. Paris: Dunod; 2002.

[47] Fontaine O, Fontaine P. Guide clinique de thérapie comportementale et cognitive. Paris: Retz; 2006.

[48] Benson H, Klipper M. The Relaxation Response. États-Unis: Avon Books; 1975

[49] Van Rillaer J. L'apprentissage de la relaxation en therapies cognitive-comportementale. Brochure d'information pour le client. Rev Fran Clin Comp Cogn 1997;2:16-23.

[50] Sniehotta FF, Scholz U, Schwarzer R. Actions plans and coping plans for physical exercise: a longitudinal intervention study in cardiac rehabilitation. Br J Health Psychol 2005;11(1):23-37.

[51] Scheeran P, Webb TL, Gollwitzer PM. The interplay between goal intentions and implementation intentions. Pers Soc Psychol Bull 2005;31:87-98.

[52] Gollwitzer PM, Scheeran P. Implementation intentions and goal achievement: a meta-analysis of effects and processes. Adv Exp Soc Psychol 2006;38:69119.

[53] Bandura A, Cervone D. Self-evaluative and self-efficacy mechanisms governing the motivational effects of goal systems. J Pers Soc Psychol 1983;45(5):1017-28.

[54] Chen HY, Chiang CK, Wang HH, Hung KY, Lee YJ, Peng YS, et al. Cognitive behavioral therapy for sleep disturbance in patients undergoing peritoneal dialysis: a pilot randomized-controlled trial. Am J Kidney Dis 2008;52(2):31423.

[55] Brown SA. Studies of educational interventions and outcomes in diabetic adults: a meta-analysis revisisted. Patient Educ Couns 1990;16(3):189-215.

[56] Prowant BF, Satalowich RJ, Murray-Bell A, Ryan LP, Schmidt LM, Kennedy JM, et al. Effectiveness of a phosphorous educational program for dialysis patients. ANNA J 1989;16(5):353-7.

[57] Shaw Stuart NJ, Stuart A. The effect of an educational patient compliance program on serum phosphate levels in patients receiving hemodialysis. J Ren Nutr 2000;10(2):80-4.

[58] Campbell KL, Ash S, Bauer JD. The impact of nutrition intervention on quality of life in pre-dialysis chronic kidney disease patients. Clin Nut 2008;27:537-44.

[59] Wingard R. Reducing early mortality in patients on dialysis: lessons from the rightstart program. J Nephrol Nurs 2009;36(2):215-20.

[60] Duarte PS, Miyazaki MC, Blay SL, Sesso R. Cognitive behavioral group therapy is an effective treatment for major depression in hemodialysis patients. Kidney Int 2009;76:414-21.

[61] Gosselin P. Le trouble d'anxiété généralisée. In: Fontaine O, Fontaine P, editors. Guide clinique de thérapie comportementale et cognitive. Paris: Retz; 2006. p. 301-44.

[62] Ladouceur R, Marchand A, Boisvert JM. Les troubles anxieux : approche cognitive et comportementale. Paris: Gaetan Morin; 1999.

[63] Ladouceur R, Bélanger L, Léger E. Arrêtez de vous faire du souci pour tout et pour rien. Paris: Odile Jacob; 2003. 